\title{
COMPARISION OF TRANSTHORACIC AND TRANSESOPHAGEAL ECHOCARDIOGRAPHY FOR THE DETECTION OF LEFT ATRIAL THROMBUS IN RHEUMATIC MITRAL VALVULAR DISEASE
}

\author{
Sharma D*, Maskey A, KC M B, Rajbhandari S*, Rajbhandari R, \\ Limbu Y R*, Malla R*, Regmi S R*, Pradhan B*, Shrestha N K
}

\section{ABSTRACT}

Transesophageal echocardiography (TEE) was performed in 114 patients with rheumatic mitral valvular disease and its results were compared with those of transthoracic echocardiography (TTE). Of 114 patients, LA thrombus was detected in 32 patients by TEE whereas TTE could detect LA thrombus in only 4 of them. Out of 32 cases, thrombus was located in left atrial appendage (LAA) in $20(62 \%)$. TTE could detect LAA thrombus in only one patient. The procedure of TEE was well tolerated and there was no complication. We conclude that TEE is superior to TTE in detection of LA cavity and LA appendage thrombus. Hence, TEE should be a routine procedure to detect LA thrombus in patients with rheumatic mitral valvular disease prior to any surgical intervention.

\section{Key Words: Left atrial appendage (LAA), Transthoracic echocardiography (TTE), Transesophageal echocardiography (TEE).}

\section{INTRODUCTION}

Detection of left atrial thrombus in patients with rheumatic mitral valvular disease is very important. It can cause recurrent systemic embolism which sometimes can be fatal and thus associated with grave prognostic significance. ${ }^{1,2}$ Presence of LA thrombus in patients with mitral stenosis precludes closed mitral commisurotomy (CMC) or percutaneous transvenous mitral commisurotomy ( PTMC). The incidence of LA thrombi in the setting of mitral valvular disease as reported in surgical data or autopsy studies, is quite significant. ${ }^{34}$ Before the advent of echocardiography, left atrial angiography was the only method available for detection of left atrial thrombi. But transseptal atrial septostomy used for left atrial angiography was associated with significant risk to the patients. ${ }^{5}$
Transthoracic two dimensional echocardiograpghy is the diagnostic technique of choice for assessment of intraatrial masses including thrombi, yet there are limitations to this approach. ${ }^{6,7}$ Although relatively large thrombi within the body of the atrium are usually evident on technically adequate thoracic images, major limitations exist in the delineation of left atrial appendage. ${ }^{6,7,8}$ Where about $50 \%$ of LA thrombi occur. ${ }^{9,10}$ Transesophageal echocardiography has helped to overcome this problem, as structure in and about left atrium, including LAA., may be readily assessed with this technique. This study describes our experience with both TTE and TEE in detection of thrombi in LA cavity and LA appendage in 114 patients with rheumatic mitral valvular disease. This is the first study of such kind carried out in Nepal.

* Shahid Gangalal National Heart Centre, Bansbari, Kathmandu, Nepal.

Address for correspondence : Dr. Deewakar Sharma

Shahid Gangalal National Heart Centre, Bansbari, Kathmandu, Nepal.

Email: sdeewakar@hotmail.com 


\section{MATERIALS AND METHODS}

The study group consisted of 114 consecutive patients with rheumatic mitral valve disease, who presented at Shahid Gangalal National Heart Centre between June 2001 and Jan 2003. Most of them had moderate to severe mitral stenosis. Their age ranged from 15-55 years (mean age 36 years). There were 74 female and 40 male patients. All the patients had transthoracic echocardiography followed by transesophageal echocardiography.

The left side of the heart in transthoracic echocardiography was imaged in apical four chamber and two chamber views as well as in parasternal long and short axis views. Modified short axis parasternal cross-sectional views at the aortic valve level were also used. The cardiac images on transesophageal echocardiography were obtained by transducer position as recommended by ASE/SCA guidelines for performing a comprehensive multiplane transesophageal examination..$^{15} 5$ MHz multiplane TEE probes (Aloka SSD 2200 \& 5500) were used for the study. The common views used were mid esophagus four chamber, two chamber, modified SAX at aortic valve level. A thrombus was defined as a mass of irregular, usually non-mobile laminated echo within the atrial cavity with a broad base attached to the wall

\section{RESULTS}

Among 114 patients studied, LA cavity and /or LAA thrombi were detected in 32 patients by TEE, whereas TTE could detect
LA thrombus in only 4 of them. Among 32 patients, 20 (62.5\%) had LAA thrombus, 8 (25\%) had thrombus in LA body cavity and $4(12.5 \%)$ had thrombi both in LAA and LA body. TTE could detect LAA thrombus in 1 patients and LA body thrombus in 3 patients. The largest thrombus was $2.6 \mathrm{~cm}$ and the smallest one was $0.9 \mathrm{~cm}$. The results and clinical data of the patients having LA/LAA thrombi are summarized in the tables below.

\section{DISCUSSION}

Although two dimensional echocardiography is considered diagnostic method of choice for detection of intracavitary masses, it has been frequently reported that this technique may fail to detect thrombi located within left atrial appendage. ${ }^{11}$ In our study, LAA thrombus could be detected in only one patient by TTE whereas TEE was able to detect LAA thrombus in 24 patients. Even 5 patients with LA cavity thrombus could not be detected by TTE and later picked by TEE. TEE could detect LA thrombus in 32 patients whereas TTE could detect thrombus only in 4 of them. In $75 \%$ of cases (24/32) thrombus was located in LAA. Hence visualization of LAA is very important. It was reported that $22 \%$ to $46 \%$ of all left atrial thrombi were confined to the appendage at the time of mitral valve surgery. ${ }^{8,9}$ Despite the use of goal directed parasternal basal short axis imaging planes, transthoracic visualization of the left atrial appendage to diagnose or exclude a thrombus is possible in only $3 \%$ to $19 \%$ of patients. ${ }^{9}$ In a study of 21 patients undergoing mitral valve replacement, TEE was $100 \%$ sensitive and specific for the diagnosis of left atrial appendage

Table I : Clinical data of 32 patients with LA thrombus

\begin{tabular}{ll|c}
\hline Age & $15-53$ \\
& Range & 35 \\
Sex & Mean & \\
& Male & 7 \\
& Female & 25 \\
Cardiac Rhythm & \\
Sinus & 2 \\
Atrial Fibrillation & 30 \\
\hline Valve Lesion & \\
MS & 28 \\
MS/MR & 2 \\
MR & 1 \\
MS/MR/AR & 1 \\
\hline
\end{tabular}

Table II : Comparative results of TTE and TEE studies

\begin{tabular}{l|c|c}
\hline \multirow{2}{*}{} & \multicolumn{2}{|c}{ No. of Patients } \\
\cline { 2 - 3 } & TTE & TEE \\
\hline Study group & 114 & 114 \\
\hline Thrombus in LA appendage & 1 & 20 \\
\hline Thrombus in LA cavity & 3 & 8 \\
\hline Thrombus in both LA cavity and LAA & - & 4 \\
\hline
\end{tabular}


thrombus. None of these thrombi were detected by transthoracic echocardiography. ${ }^{12}$ In a series of 2000 consecutive patients undergoing TEE for a wide variety of clinical indications, the incidence of left atrial thrombus was $3.8 \%$; all of these were undetected by transthoracic echocardiography. Thrombus within LA appendage was detected in $100 \%$ of patients by TEE and missed in all patients by transthoracic study. ${ }^{13,14}$ Majority of the patients in our study had moderate to severe mitral stenosis and were in atrial fibrillation. Atrial fibrillation is a known predisposing factor for left atrial appendage thrombus formation.

\section{LIMITATIONS}

This is a small study comprising 114 patients only. Only 9 patients had open heart surgery and thrombus was verified in all these patients. Rest of the patients with LA thrombus are on oral anticoagulation with periodic follow up.

\section{CONCLUSION}

Because of the immediate proximate anatomic relation of the left atrium to the esophagus, TEE allows outstanding high resolution delineation of the body and appendage of the left atrium. By providing high resolution images in multiple planes, TEE is far superior to transthoracic echocardiography in detection of LA cavity/ LA appendage thrombus. Hence, TEE should be a routine procedure to detect LA appendage clot in patients with rheumatic mitral valve disease, especially in dominant mitral stenosis, prior to PTMC or CMC.

\section{REFERENCES}

1. Schwarts SP, Biloon S: The clinical signs of occluding thrombi of left auricle. Am Heart J7:84,1931

2. Kossowsky WA, Bleifer SB. Fatal cerebral embolus complicating transseptal left heart Catheterization, Circulation 32:811, 1965

3. Jordan NA, Schiefly CH, Edwards JE. Mural thrombus and arterial embolism in mitral stenosis. Circulation, 3;363, 1951

4. Bansal RC, Heywaood JT, Applegate PM, Jutzy KR. Detection of left atrial thrombi by two dimensional echocardiography and surgical correlation in 148 patients with mitral valve disease. Am J Cardiol 63:243, 1989

5. BraunwaldE: Transseptal left heart Catheterization: Circulation 37 (Suppl III) : III-74,1968

6. Schweizer P, Bardos F, Erbel R et al; Detection of left atrial thrombus by Echocardiography. Br Heart $J$, 1981;45: 14856

7. Baker KM, Martin RP. Two dimensional echocardiographic detection of left atrial thrombi in Rheumatic mitral valve disease (abstract) . J Am Coll Cardiol 1983; 1:1:703

8. Shrestha NK, Moreno FL, Narciso FV et al. Two dimensional echocardiographic diagnosis of left atrial thrombus in Rheumatic heart disease. A clinicopathologic study. Circulation $1983 ; 67: 341-7$

9. Heroz CA, Bass D, Kane M, Asinger R. Two dimensional echocardiographic imaging of left atrial appendage thrombi. $J$ Am Coll Cardiol 1984; 3:1340-4

10. A Sreenivasa Rao, R.S.Murthy, P.Bhaskar Naida, K.Raghu, A.V.Anjaneyulu. P. Krishnam Raju, Sudhir R. Naik. Transesophageal Echocardiography for the detection of left atrial thrombus. Indian Heart Journal, $1994 ; 46 ; 1 ; 37-40$

11. Come PC, Rity MF, Markins JE, Malagold M. Limitations of echocardiographic techniques in evaluation of left atrial masses. J Am Coll Cardiol 3:1340, 1984

12. Aschenberg $W$, Schluter $M$, Kremer $P$, Schroder $E$, Siglow $V$, Bleifeld $W$, Transesophageal two dimensional echocardiography for the detection of left atrial appendage thrombus. J Am coll Cordiol 1986; 7:163-6

13. Mugg A, Daniel WG, Hansmann D et al, Diagnosis of left atrial thrombi by transesophageal echocardiography. Clinical implications and follow up. Am J card imaging 1990;4:173-9.

14. Mugge A, Daniel WG, Hevench A, Lichtlen PR. Diagnosis of non-infective mass lesions by two dimensional echocardiography. Comparision of the transthoracic and transesophageal approaches. Circulation 1991;83:70-8.

15. Shanewise J S, Cheung AT, Aronson S, Stewari W J, Weiss R L, Mark BJ, Savage R M, et al. ASE/SCA Guidelines for performing a comprehensive intraoperative multiplane transesphageal Echocardigraphic examination. Recommendation of the American Society of Echocardiography council for intraoperative Echocardiography and the Society of cardiovascular anaesthesiologists task force for certification in perioperative transoesophageal Echocardiography. Cardovascular anaesthesia.1999; 89: 870-84.

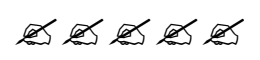

